\title{
No-bend Orthogonal Drawings of Series-Parallel Graphs (Extended Abstract)
}

\author{
Md. Saidur Rahman ${ }^{1}$, Noritsugu Egi ${ }^{2}$, and Takao Nishizeki ${ }^{2}$ \\ 1 Department of Computer Science and Engineering, \\ Bangladesh University of Engineering and Technology (BUET), \\ Dhaka 1000, Bangladesh \\ saidurrahman@cse.buet.ac.bd \\ 2 Graduate School of Information Sciences, Tohoku University, Aoba-yama 05, \\ Sendai 980-8579, Japan \\ egi@nishizeki.ecei.tohoku.ac.jp, nishi@ecei.tohoku.ac.jp
}

\begin{abstract}
In a no-bend orthogonal drawing of a plane graph, each vertex is drawn as a point and each edge is drawn as a single horizontal or vertical line segment. A planar graph is said to have a no-bend orthogonal drawing if at least one of its plane embeddings has a no-bend orthogonal drawing. Every series-parallel graph is planar. In this paper we give a linear-time algorithm to examine whether a series-parallel graph $G$ of the maximum degree three has a no-bend orthogonal drawing and to find one if $G$ has.
\end{abstract}

Keywords: Planar Graph, Algorithm, Graph Drawing, Orthogonal Drawing, Bend, SPQ tree.

\section{Introduction}

An orthogonal drawing of a planar graph $G$ is a drawing of $G$ such that each vertex is mapped to a point, each edge is drawn as a sequence of alternate horizontal and vertical line segments, and any two edges do not cross except at their common end [NR04, RN02, RNN99, T87. A bend is a point where an edge changes its direction in a drawing. If $G$ has a vertex of degree five or more, then $G$ has no orthogonal drawing. On the other hand, if $G$ has no vertex of degree five or more, that is, the maximum degree $\Delta$ of $G$ is at most four, then $G$ has an orthogonal drawing, but may need bends. Minimization of the number of bends in an orthogonal drawing is a challenging problem. A bendminimum orthogonal drawing of a planar graph $G$ has the minimum number of bends among all possible planar orthogonal drawings of $G$. The problem of finding a bend-minimum orthogonal drawing is one of the most famous problems in the graph drawing literature [BEGKLM04 and has been studied both in the fixed embedding setting RN02, RNN03, RNN99, T87 and in the variable embedding setting DLV98, GT01. Some plane graphs with fixed embeddings have an orthogonal drawing without bends, in which each edge is drawn by a 


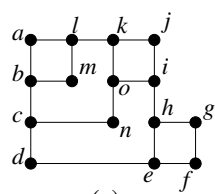

(a)

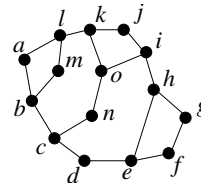

(b)

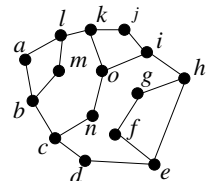

(c)

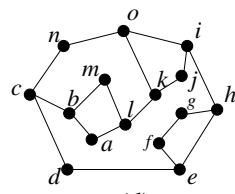

(d)

Fig. 1. (a) A no-bend drawing, and (b)-(d) three embeddings of the same planar graph

single horizontal or vertical line segment RNN03. We call such a drawing a no-bend drawing of a plane graph. Figure 1(a) depicts a no-bend drawing of the plane graph in Fig. 1(b). As a result in the fixed embedding, Rahman et al. RNN03 obtained a necessary and sufficient condition for a plane graph $G$ of $\Delta \leq 3$ to have a no-bend drawing, and gave a linear-time algorithm to find a no-bend drawing if $G$ has.

We say that a planar graph $G$ has a no-bend drawing if at least one of the plane embedding of $G$ has a no-bend drawing. Figures 1(b), (c) and (d) depict three of all plane embeddings of the same planar graph $G$. Among them only the embedding in Fig. 11(b) has a no-bend drawing as illustrated in Fig. 1(a). Thus the planar graph $G$ has a no-bend drawing. It is an NP-complete problem to examine whether a planar graph $G$ of $\Delta \leq 4$ has a no-bend drawing in the variable embedding setting GT01. However, for a planar graph $G$ of $\Delta \leq 3$, Di Battista et al. DLV98 gave an $O\left(n^{5} \log n\right)$ time algorithm to find a bendminimum orthogonal drawing of $G$. Every series-parallel graph is a planar graph, and their algorithm takes time $O\left(n^{3}\right)$ for a series-parallel graph with $\Delta \leq 3$. Thus, by their algorithm one can examine in time $O\left(n^{3}\right)$ whether a series-parallel graph with $\Delta \leq 3$ has a no-bend drawing. As another result in the variable embedding, Rahman et al. REN05 gave a linear time algorithm to examine whether a subdivision $G$ of a planar triconnected cubic graph has a no-bend drawing, and to find a no-bend drawing of $G$ if $G$ has.

In this paper we study the problem of no-bend orthogonal drawings of seriesparallel graphs with $\Delta \leq 3$ in the variable embedding setting, and give a linear algorithm to find a no-bend orthogonal drawing if $G$ has.

The rest of the paper is organized as follows. Section 2 describes some definitions and presents preliminary results. Section 3 presents our algorithm to find a no-bend drawing of a biconnected series-parallel graph $G$ if $G$ has. Finally Section 4 is a conclusion.

\section{Preliminaries}

In this section we give some definitions and present preliminary results.

Let $G=(V, E)$ be a connected graph with vertex set $V$ and edge set $E$. The degree $d(v)$ of a vertex $v$ is the number of edges incident to $v$ in $G$. We denote the maximum degree of graph $G$ by $\Delta(G)$ or simply by $\Delta$. The connectivity $\kappa(G)$ of a graph $G$ is the minimum number of vertices whose removal results in a disconnected graph or a single-vertex graph $K_{1}$. We say that $G$ is $k$-connected if $\kappa(G) \geq k$. 
A graph $G=(V, E)$ is called a series-parallel graph (with source $s$ and sink $t$ ) if either $G$ consist of a pair of vertices connected by a single edge, or there exist two series-parallel graphs $G_{i}=\left(V_{i}, E_{i}\right), i=1,2$, with source $s_{i}$ and sink $t_{i}$ such that $V=V_{1} \cup V_{2}, E=E_{1} \cup E_{2}$, and either $s=s_{1}, t_{1}=s_{2}$ and $t=t_{2}$ or $s=s_{1}=s_{2}$ and $t=t_{1}=t_{2}$.

A pair $\{u, v\}$ of vertices of a connected graph $G$ is a split pair if there exist two subgraphs $G_{1}=\left(V_{1}, E_{1}\right)$ and $G_{2}=\left(V_{2}, E_{2}\right)$ satisfying the following two conditions: 1. $V=V_{1} \cup V_{2}, V_{1} \cap V_{2}=\{u, v\}$; and 2. $E=E_{1} \cup E_{2}, E_{1} \cap E_{2}=\emptyset$, $\left|E_{1}\right| \geq 1,\left|E_{2}\right| \geq 1$. Thus every pair of adjacent vertices is a split pair. A split component of a split pair $\{u, v\}$ is either an edge $(u, v)$ or a maximal connected subgraph $H$ of $G$ such that $\{u, v\}$ is not a split pair of $H$. A split pair $\{u, v\}$ of $G$ is called a maximal split pair with respect to a reference split pair $\{s, t\}$ if, for any other split pair $\left\{u^{\prime}, v^{\prime}\right\}$, vertices $s, t, u$ and $v$ are in the same split component of $\left\{u^{\prime}, v^{\prime}\right\}$.

Let $G$ be a biconnected series-parallel graph. Let $(s, t)$ be an edge of $G$. The SPQ-tree $\mathcal{T}$ of $G$ with respect to a reference edge $e=(s, t)$ describes a recursive decomposition of $G$ induced by its split pairs GL99. Tree $\mathcal{T}$ is a rooted ordered tree whose nodes are of three types: $S, P$ and $Q$. Each node $x$ of $\mathcal{T}$ corresponds to a subgraph of $G$, called its pertinent graph $G_{x}$. Each node $x$ of $\mathcal{T}$ has an associated biconnected multigraph, called the skeleton of $x$ and denoted by skeleton $(x)$. Tree $\mathcal{T}$ is recursively defined as follows.

- Trivial Case: In this case, $G$ consists of exactly two parallel edges $e$ and $e^{\prime}$ joining $s$ and $t$. $\mathcal{T}$ consists of a single $Q$-node $x$. The skeleton of $x$ is $G$ itself. The pertinent graph $G_{x}$ consists of only the edge $e^{\prime}$.

- Parallel Case: In this case, the split pair $\{s, t\}$ has three or more split components $G_{0}, G_{1}, \cdots, G_{k}, k \geq 2$, and $G_{0}$ consists of only a reference edge $e=(s, t)$. The root of $\mathcal{T}$ is a $P$-node $x$. The skeleton $(x)$ consists of $k+1$ parallel edges $e_{0}, e_{1}, \cdots, e_{k}$ joining $s$ and $t$. The pertinent graph $G_{x}=G_{1} \cup G_{2} \cup \cdots \cup G_{k}$ is a union of $G_{1}, G_{2}, \cdots, G_{k}$. (The skeleton of $P$-node $p_{2}$ in Fig. 2 consists of three parallel edges joining vertices $e$ and $g$. Figure 2(e) depicts the pertinent graph of $p_{2}$.)

- Series Case: In this case the split pair $\{s, t\}$ has exactly two split components, and one of them consists of the reference edge $e$. One may assume that the other split component has cut-vertices $c_{1}, c_{2}, \cdots, c_{k-1}, k \geq 2$, that partition the component into its blocks $G_{1}, G_{2}, \cdots, G_{k}$ in this order from $s$ to $t$. Then the root of $\mathcal{T}$ is an $S$-node $x$. The skeleton of $x$ is a cycle $e_{0}, e_{1}, \cdots, e_{k}$ where $e_{0}=e$, $c_{0}=s, c_{k}=t$, and $e_{i}$ joins $c_{i-1}$ and $c_{i}, 1 \leq i \leq k$. The pertinent graph $G_{x}$ of node $x$ is a union of $G_{1}, G_{2}, \cdots, G_{k}$. (The skeleton of $S$-node $s_{2}$ in Fig. 2 is the cycle $c, d, e, g, h, a, c$. Figure 2(d) depicts the pertinent graph $G_{s_{2}}$ of $s_{2}$.)

In all cases above, we call the edge $e$ the reference edge of node $x$. Except for the trivial case, node $x$ of $\mathcal{T}$ has children $x_{1}, x_{2}, \cdots, x_{k}$ in this order; $x_{i}$ is the root of the SPQ-tree of graph $G_{i} \cup e_{i}$ with respect to the reference edge $e_{i}, 1 \leq i \leq k$. We call edge $e_{i}$ the reference edge of node $x_{i}$, and call the endpoints of edge $e_{i}$ the poles of node $x_{i}$. The tree obtained so far has a $Q$-node associated with each 


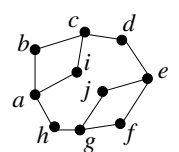

(a)

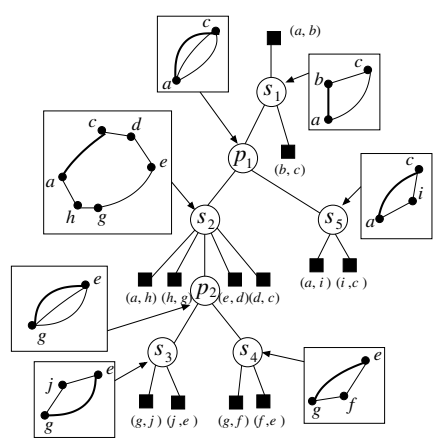

(b)

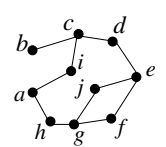

(c)

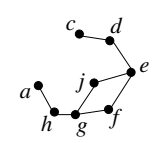

(d)

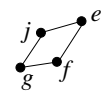

(e)

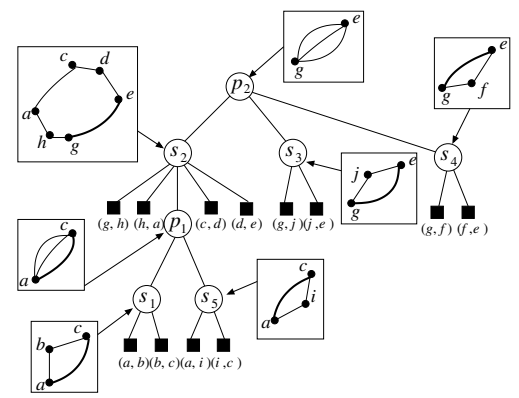

(f)

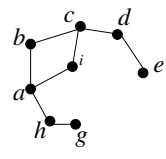

(g)

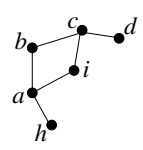

(h)

Fig. 2. (a)A biconnected series-parallel graph $G$ with $\Delta=3$, (b) SPQ-tree $\mathcal{T}$ of $G$ with respect to reference edge $(a, b)$, and skeletons of $P$ - and $S$-nodes, (c) the pertinent graph $G_{s_{1}}$ of $S$-node $s_{1}$, (d) the pertinent graph $G_{s_{2}}$ of $S$-node $s_{2}$, (e) the pertinent graph $G_{p_{2}}$ of $P$-node $p_{2}$, (f) SPQ-tree $\mathcal{T}$ of $G$ with $P$-node $p_{2}$ as the root, (g) the pertinent graph of $S$-node $s_{2}$, and (h) the core graph of $s_{2}$

edge of $G$, except the reference edge $e$. We complete the SPQ-tree $\mathcal{T}$ by adding a $Q$-node, representing the reference edge $e$, and making it the parent of $x$ so that it becomes the root of $\mathcal{T}$. An example of the SPQ-tree of a biconnected series-parallel graph in Fig. 2(a) is illustrated in Fig. 2(b), where the edge drawn by a thick line in each skeleton is the reference edge of the skeleton.

The SPQ-tree $\mathcal{T}$ defined above is a special case of an "SPQR-tree" DT96. GL99] where there is no $R$-node and the root of the tree is a $Q$-node corresponding to the reference edge $e$. One can easily modify $\mathcal{T}$ to an SPQ-tree $\mathcal{T}^{\prime}$ with an arbitrary $P$-node as the root as illustrated in Fig. 2 (f).

In the remainder of this paper, we thus consider a SPQ-tree $\mathcal{T}$ with a $P$-node as the root. If $\Delta=2$, then a biconnected series-parallel graph $G$ is a cycle, and a cycle $G$ has a no-bend drawing if and only if $G$ has four or more vertices. One may thus assume that $\Delta \geq 3$, and that the root $P$-node of $\mathcal{T}$ has three or more children. Then the pertinent graph $G_{x}$ of each node $x$ is the subgraph of $G$ induced by the edges corresponding to all descendant $Q$-node of $x$. The following facts can be easily derived from the fact that each vertex of $G$ has degree at most three and $G$ has no multiple edges. 
Fact 1. Let $(s, t)$ be the reference edge of an $S$-node $x$ of $\mathcal{T}$, and let $x_{1}, x_{2}, \cdots, x_{k}$ be the children of $x$ in this order from $s$ to $t$. Then (i) each child $x_{i}$ of $x$ is either a $P$-node or a $Q$-node; (ii) both $x_{1}$ and $x_{k}$ are $Q$-nodes; and (iii) $x_{i-1}$ and $x_{i+1}$ must be $Q$-nodes if $x_{i}$ is a $P$-node where $2 \leq i \leq k-1$.

Fact 2. Each non-root $P$-node of $\mathcal{T}$ has exactly two children, and either both of the two children are $S$-nodes or one of them is an $S$-node and the other is a $Q$-node.

Let $x$ be an $S$-node of $\mathcal{T}$, and let $u$ and $v$ be the poles of the pertinent graph of $x$. Let $x_{1}, x_{2}, \cdots, x_{k}$ be the children of $x$ in this order from $u$ to $v$. From Fact $1 x_{1}$ and $x_{k}$ are $Q$-nodes. Thus $x_{1}$ and $x_{k}$ correspond to edges $\left(u, u^{\prime}\right)$ and $\left(v^{\prime}, v\right)$ of $G$, respectively. Then the core graph for $x$ is a graph obtained from the pertinent graph of $x$ by deleting vertices $u$ and $v$. (Figure 2 (g) illustrates a pertinent graph of S-node $s_{1}$ for $\mathcal{T}$ in Fig. 2(f), and Fig. 2(h) illusrates a core graph for $s_{1}$.) Vertices $u^{\prime}$ and $v^{\prime}$ are called the poles of the core graph for $x$, and edges $\left(u, u^{\prime}\right)$ and $\left(v^{\prime}, v\right)$ are called hands of the core graph for $x$. (In Figs. 2(g) and (h) the poles of the core graph of $S$-node $s_{1}$ are vertices $d$ and $h$.) For a $P$ or $Q$-node $x$ in $\mathcal{T}$, we define the core graph for $x$ as the pertinent graph of $x$, and the poles of the core graph for $x$ is the same as the poles of the pertinent graph of $x$. The core graph of a $P$ - or $Q$-node has no hand.

A drawing of a planar graph $G$ is called an orthogonal drawing of $G$ if each vertex is mapped to a point, each edge is drawn as a sequence of alternate horizontal and vertical line segments, and any two edges do not cross except at their common end. We call an orthogonal drawing $D$ of $G$ a no-bend drawing if $D$ has no bend, that is, each edge is drawn as a single horizontal or vertical line segment. A polar drawing of a series-parallel graph $G$ is a no-bend drawing of $G$ in which the two poles $u$ and $v$ of $G$ are drawn on the outer face $F_{o}$ of the drawing.

We call a polar drawing $D$ of a series-parallel graph $G$ a diagonal drawing if $D$ intersects neither the first quadrant with the origin at pole $u$ nor the third quadrant with the origin at pole $v$ after rotating the drawing and renaming the poles if necessary, as illustrated in Fig. 3(a). Throughout the paper a quadrant is considered to be a closed plane region. Both a drawing of a single vertex as a point and a drawing of a single edge as a straight line-segment are diagonal drawings.

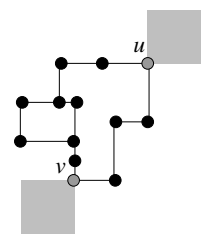

(a)

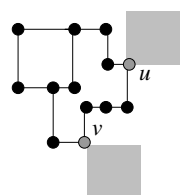

(b)

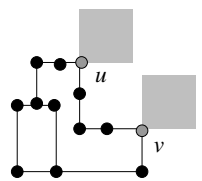

(c)

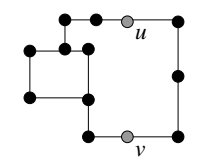

(d)

Fig. 3. Polar drawings of a graph $G$ with poles $u$ and $v$ : (a) a diagonal drawing, (b) a side-on drawing, (c) an L-shape drawing, (d) another polar drawing 
We call a polar drawing $D$ of $G$ a side-on drawing if $D$ intersects neither the first quadrant with the origin at $u$ nor the fourth quadrant with the origin at $v$ after rotating the drawing and renaming the poles if necessary, as illustrated in Fig. 3(b). A drawing of a single vertex as a point is regarded not to be a side-on drawing, while a drawing of a single edge as a straight line-segment is a side-on drawing.

A polar drawing $D$ is called an $L$-shape drawing if $D$ intersects neither the first quadrant with the origin at $u$ nor the first quadrant with the origin at $v$ after rotating the drawing and renaming the poles if necessary, as illustrated in Fig. 3(c). A drawing of a single vertex as a point is regarded not to be an L-shape drawing. A drawing of a single edge as a straight line-segment is not an L-shape drawing.

We say that a polar drawing is good if it is a diagonal, side-on or L-shape drawing. Not every polar drawing $D$ is good. For example, the polar drawing in Fig. 3(d) is not good, because it is not a diagonal, side-on drawing or L-shape drawing.

In the next section we give an algorithm for constructing no-bend drawing of a biconnected series-parallel graph $G$ with $\Delta=3$.

Our idea is as follows. Let $\mathcal{T}$ be an SPQ-tree of $G$. The core graph of each leaf-node of $\mathcal{T}$ consists of a single edge. For each leaf-node of $\mathcal{T}$ we first draw the core graph by a line segment as a diagonal or side-on drawing. Then, in bottom up fashion, we find a diagonal drawing, a side-on drawing, and an Lshape drawing of the core graph for each internal node $x$ of $\mathcal{T}$ by merging the drawings corresponding to the children of $x$ if they exist. The drawing of the graph corresponding to the root-node of $\mathcal{T}$ yields a no-bend drawing of $G$ if $G$ has a polar drawing with the split pair, corresponding to the root $P$-node, as the poles. Our algorithm eventually chooses an appropriate SPQ-tree $\mathcal{T}$ of $G$ such that the drawing of a plane graph corresponding to the root-node of $\mathcal{T}$ yields a no-bend drawing of $G$ if $G$ has. (See Fig. 8 for illustration.)

As we see later, we construct a no-bend drawing of the core graph for a node $x$ in $\mathcal{T}$ by merging the no-bend drawings of the core graphs for the childrens of $x$; the no-bend drawing of the core graph for each children of $x$ must be a polar drawing with the two poles of the core graph. A side-on drawing is found more suitable for merging than a diagonal drawing, and an L-shape drawing is found more suitable for merging than a side-on drawing. Intuitively, to connect the two poles by a sequence of horizontal and vertical line segments, at least three turns are required for a diagonal drawing, at least two turns are required for a side-on drawing and only one turn is required for an L-shape drawing. A graph may have a diagonal drawing although it has no side-on or L-shape drawing and a graph may have a side-on drawing although it has no L-shape drawing. We call a polar drawing $D$ of a core graph $H(x)$ for a node $x$ in $\mathcal{T}$ a desirable drawing if one of the following (a), (b) and (c) holds: (a) $D$ is an L-shape drawing; (b) $D$ is a side-on drawing, and $H(x)$ has no L-shape drawing; (c) $D$ is a diagonal drawing, and $H(x)$ has neither an L-shape drawing nor a side-on drawing. Throughout the paper we denote by $D(x)$ a desirable drawing of the core graph $H(x)$ for a node $x$ in $\mathcal{T}$. 


\section{No-bend Drawings of Biconnected Series-Parallel Graphs}

In this section we give an algorithm to construct a no-bend orthogonal drawing of a biconnected series-parallel graph $G$ whenever $G$ has.

If $G$ is a cycle, then it is easy to find a no-bend drawing of $G$; $G$ has a no-bend drawing if and only if $G$ has four or more vertices. We thus assume that $G$ is not a cycle.

Let $\mathcal{T}$ be an SPQ-tree of $G$ whose root is a $P$-node $x_{p}$ having three children. (See Fig. 2(f).) We now have the following lemma.

Lemma 3. Let $G$ be a series-parallel graph with $\Delta \leq 3$, let $\mathcal{T}$ be an $S P Q$-tree with a P-node $x_{p}$ as the root, and let $x$ be a non-root node in $\mathcal{T}$. If the core graph $H(x)$ of $x$ has a no-bend drawing, then the following (a) and (b) hold: (a) $H(x)$ has a side-on or diagonal drawing, and hence $H(x)$ has a desirable drawing $D(x)$; and (b) if a desirable drawing of $H(x)$ is a diagonal drawing, then every no-bend drawing of $H(x)$ is a diagonal drawing for the poles of $H(x)$.

Proof. We will prove the claim by induction based on $\mathcal{T}$.

We first assume that $x$ is a leaf-node, that is, a $Q$-node. In this case $H(x)$ consists of a single edge $e=(u, v)$, and $u$ and $v$ are the poles of $H(x)$. We thus draw $e$ as a single vertical line segment, which is a side-on drawing $D(x)$ of $H(x)$. Since $H(x)$ has no L-shape drawing, $D(x)$ is a desirable drawing. Thus (a) and (b) hold.

We next assume that $x$ is an inner node other than the root $x_{p}$ and that $H(x)$ has a no-bend drawing. Let $u$ and $v$ are the poles of $H(x)$. Let $x_{1}, x_{2}, \cdots, x_{k}$ $(k \geq 2)$ be the children of $x$ in this order from $u$ to $v$. Since $H(x)$ has a no-bend drawing, each $H\left(x_{i}\right)$ has a no-bend drawing. Thus we suppose inductively that (a) and (b) hold for each child of $x$. We now have two cases to consider.

Case 1: $x$ is an $S$-node.

Suppose that $x$ has exactly two children. Then $H(x)$ consists of a single vertex. We draw $H(x)$ as a point. Then the diagonal drawing is a desirable drawing $D(x)$. Thus (a) and (b) hold.

We thus assume that $x$ has exactly $k$ children and $k \geq 3$. Then $H(x)=$ $H\left(x_{2}\right) \cup H\left(x_{3}\right) \cup \cdots \cup H\left(x_{k-1}\right)$, where $H\left(x_{i}\right)$ is the core graph of $x_{i}$. The hypothesis implies that, for each $i, 2 \leq i \leq k-1$, (a) and (b) hold for the core graph $H\left(x_{i}\right)$. We now have the following four subcases to consider.

Case 1(a): $k=3$.

In this case $H(x)=H\left(x_{2}\right)$, hence (a) and (b) hold for $H(x)$. Case 1(b): $k=4$.

In this case $H(x)=H\left(x_{2}\right) \cup H\left(x_{3}\right)$. Fact 1(iii) implies that either both $x_{2}$ and $x_{3}$ are $Q$-nodes or one of them is a $P$-node and the other one is a $Q$-node.

If $x_{2}$ and $x_{3}$ are $Q$-nodes, then we can construct both an L-shape drawing and a side-on drawing of $H(x)$, as illustrated in Figs. 4(a) and 5(a). Thus a desirable drawing of $H(x)$ is an L-shape drawing, and hence (a) and (b) hold. We thus assume that one of them, say $x_{2}$, is a $P$-node and the other is a $Q$-node. 


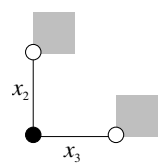

(a)

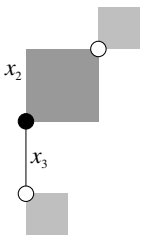

(b)

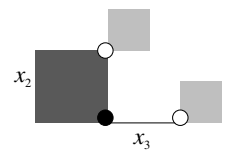

(c)

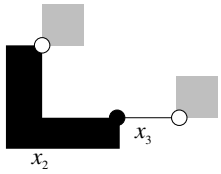

(d)

Fig. 4. Desirable drawings of the core graph for $S$-nodes with four children

We first consider the case where a desirable drawing $D\left(x_{2}\right)$ of $H\left(x_{2}\right)$ is a diagonal drawing. In this case we can construct a side-on drawing $D(x)$ of $H(x)$ as illustrated in Fig. 4(b). Since the desirable drawing of $H\left(x_{2}\right)$ is a diagonal drawing, $H\left(x_{2}\right)$ has neither an L-shape drawing nor a side-on drawing, and hence clearly $H(x)$ has no L-shape drawing. Therefore the side-on drawing $D(x)$ of $H(x)$ is a desirable drawing. Hence (a) and (b) hold.

We next consider the case where the desirable drawing $D\left(x_{2}\right)$ of $H\left(x_{2}\right)$ is a side-on drawing. Then we can construct both an L-shape drawing $D(x)$ and a side-on drawing of $H(x)$ as illustrated in Figs. 4(c) and 5(c). Hence (a) and (b) hold.

We finally consider the case where the desirable drawing $D\left(x_{2}\right)$ of $H\left(x_{2}\right)$ is an L-shape drawing. Then we can construct an L-shape drawing $D(x)$ of $H(x)$ as illustrated in Fig. $4(\mathrm{~d})$. $H\left(x_{2}\right)$ has a side-on or diagonal drawing. From it one can easily construct a side-on drawing of $H(x)$ as illustrated in Figs. 5 (b) and (c). Therefore (a) and (b) hold.

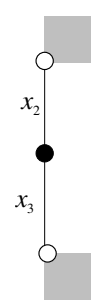

(a)

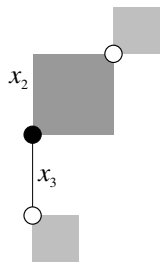

(b)

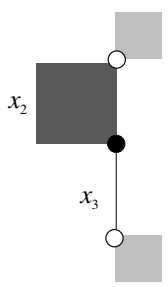

(c)

Fig. 5. Side-on drawings of the core graph for $S$-nodes with four children

Case 1(c): $k=5$.

In this case, $H=H\left(x_{2}\right) \cup H\left(x_{3}\right) \cup H\left(x_{4}\right)$. Fact 1(iii) implies that at least one of $x_{2}, x_{3}$ and $x_{4}$ is a $Q$-node. In this case we can construct a no-bend drawing of $H(x)$ such that (a) and (b) hold. The details are omitted in this extended abstract.

Case $1(d): k \geq 6$.

In this case $H=H\left(x_{2}\right) \cup H\left(x_{3}\right) \cup \cdots \cup H\left(x_{k-1}\right), k \geq 6$. Fact 1 (iii) implies that there are two or more $Q$-nodes among $x_{2}, x_{3}, \cdots x_{k-1}$. Therefore we can easily 
construct both an L-shape drawing and a side-on drawing $D$ of $H(x)$, and hence (a) and (b) hold.

Case 2: $x$ is a $P$-node.

In this case $k=2$ and $x$ has exactly two children $x_{1}$ and $x_{2}$. Then the hypothesis implies that, for $i=1,2$, (a) and (b) hold for $H\left(x_{i}\right)$. By Fact 2 either both $x_{1}$ and $x_{2}$ are $S$-nodes or one of $x_{1}$ and $x_{2}$ is an $S$-node and the other is a $Q$-node. We first assume that one of $x_{1}$ and $x_{2}$, say $x_{1}$, is a $Q$-node, then we have the following two subcases.

Case 2(a): The desirable drawing $D\left(x_{2}\right)$ of $H\left(x_{2}\right)$ is a diagonal drawing.

In this case $H\left(x_{2}\right)$ has neither an L-shape drawing nor a side-on drawing. Furthermore, every no-bend drawing of $H\left(x_{2}\right)$ is a diagonal drawing by induction hypothesis. Then $D\left(x_{1}\right), D\left(x_{2}\right)$ and the drawings of hands of $H\left(x_{2}\right)$ cannot be merged without bends as illustrated in Fig. 6(a). Therefore $H(x)$ does not have a no-bend drawing, contrary to the assumption that $H(x)$ has a no-bend drawing. Therefore this case does not occur.

Case 2(b): The desirable drawing $D\left(x_{2}\right)$ of $H\left(x_{2}\right)$ is a side-on or L-shape drawing.

In this case we can construct a no-bend drawing $D(x)$ of $H(x)$ such that (a) and (b) hold as illustrated in Figs. 6(b)-(i).

$\mathcal{Q} . \mathcal{E} . \mathcal{D}$.

We call the algorithm described in the proof of Lemma 3 for finding a desirable drawing $D(x)$ of $H(x)$ Algorithm Desirable-Drawing whenever $H(x)$ has a nobend drawing. Clearly Algorithm Desirable-Drawing takes linear-time.

In the rest of the section we give Algorithm Biconnected-Draw for finding a no-bend drawing of $G$ whenever $G$ has. Remember that the root node $x_{p}$ in $\mathcal{T}$

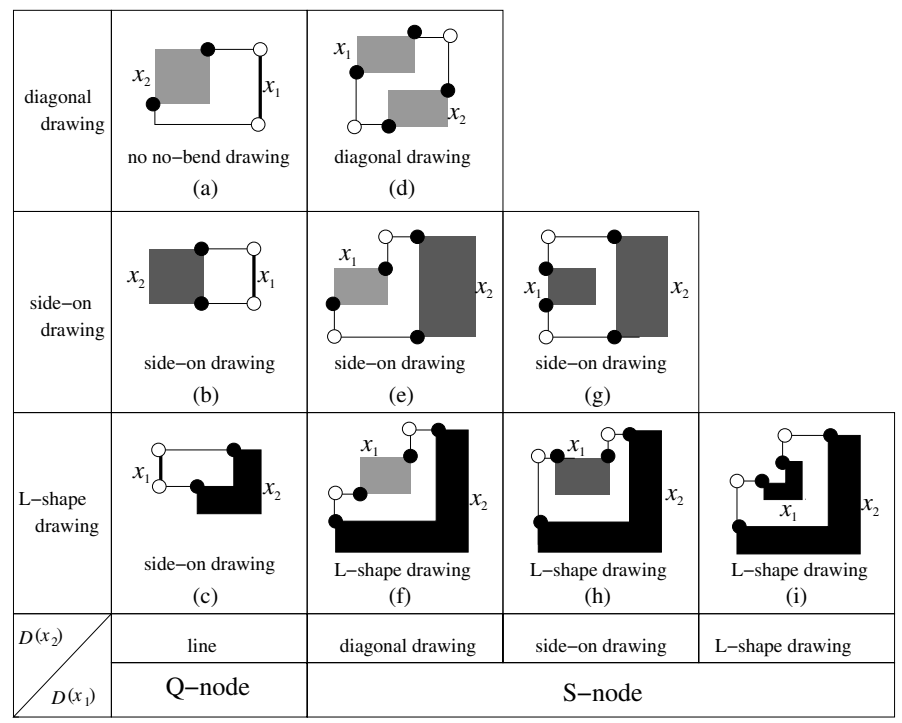

Fig. 6. Drawings of $H(x)$ for a $P$-node $x \neq x_{p}$ 
has three children as depicted in Fig. $2(\mathrm{f})$. Let $x_{1}, x_{2}$ and $x_{3}$ be the three children of $x_{p}$ in $\mathcal{T}$. If $G$ has a no-bend drawing, then $H\left(x_{i}\right), 1 \leq i \leq 3$, has a no-bend drawing. For $1 \leq i \leq 3$, we find a desirable drawing $D\left(x_{i}\right)$ of $H\left(x_{i}\right)$ by Algorithm Desirable-Drawing. If $G$ has a polar drawing for the poles corresponding to $x_{p}$, then we now find a no-bend drawing of $G=H\left(x_{p}\right)$ by merging the drawings of $D\left(x_{1}\right), D\left(x_{2}\right), D\left(x_{3}\right)$ and the drawings of their hands. Otherwise, we find appropriate poles for which $G$ has a no-bend polar drawing. Since $G$ is a simple graph, at most one of $x_{1}, x_{2}$ and $x_{3}$ is a $Q$-node. We now have the following two cases to consider.

Case 1: one of them, say $x_{3}$, is a $Q$-node.

In this case only $x_{3}$ is a $Q$-node. If at least one of $D\left(x_{1}\right)$ and $D\left(x_{2}\right)$ is a diagonal drawing, Then $G$ does not have a no-bend drawing as illustrated in Fig. [7(a)-(c). Otherwise, $G$ has a no-bend drawing as illustrated in Fig. 7(d)-(f). The details are omitted.

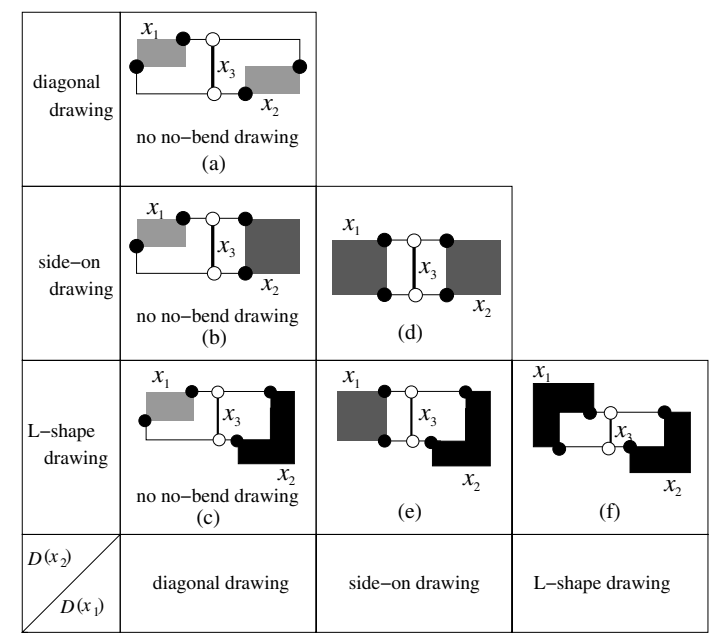

Fig. 7. Illustration for Case 1 of Algorithm Biconnected-Draw

Case 2: all of $x_{1}, x_{2}$ and $x_{3}$ are $S$-nodes.

If at most one of $D\left(x_{1}\right), D\left(x_{2}\right)$ and $D\left(x_{3}\right)$ is a diagonal drawing, then we can easily construct a no-bend drawing of $G$. If all of $D\left(x_{1}\right), D\left(x_{2}\right)$ and $D\left(x_{3}\right)$ are diagonal drawings, then one can easily observe that $G$ does not have a no-bend drawing.

We thus consider the case where exactly two of $D\left(x_{1}\right), D\left(x_{2}\right)$ and $D\left(x_{3}\right)$ are diagonal drawings. If two of $D\left(x_{1}\right), D\left(x_{2}\right)$ and $D\left(x_{3}\right)$ are diagonal drawings and the other is an L-shape drawing, then clearly we can construct a no-bend drawing of $G$. We may thus assume that two of $D\left(x_{1}\right), D\left(x_{2}\right)$ and $D\left(x_{3}\right)$ are diagonal drawings and the other is a side-on drawing.

We may assume without loss of generality that $D\left(x_{1}\right)$ and $D\left(x_{2}\right)$ are diagonal drawings and $D\left(x_{3}\right)$ is a side-on drawing. By Lemma 3 (b) every no-bend drawing of each of $H\left(x_{1}\right)$ and $H\left(x_{2}\right)$ is a diagonal drawing. By merging $D\left(x_{1}\right)$ and $D\left(x_{2}\right)$ 


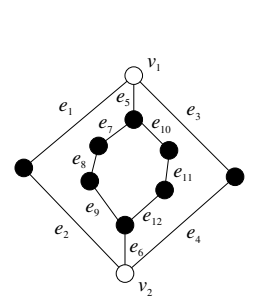

(a)

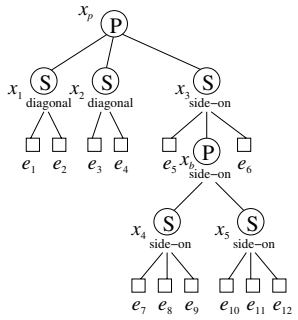

(b) $\mathcal{T}$

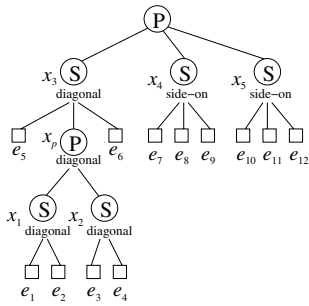

(f) $\mathcal{T}^{\prime}$

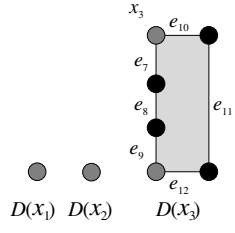

(c)

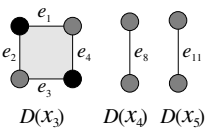

(g)

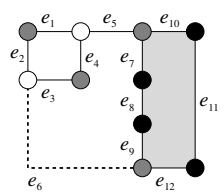

(d)

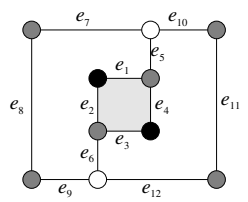

(h)

Fig. 8. (a) - (d) A no-bend drawing of $G$ cannot be found using tree $\mathcal{T}$, and (e)-(h) a no-bend drawing of $G$ can be found using tree $\mathcal{T}^{\prime}$

we can obtain only a diagonal drawing $D^{\prime}$. Since $D\left(x_{3}\right)$ is a side-on drawing, $D^{\prime}$ and $D\left(x_{3}\right)$ cannot be merged to produce a no-bend drawing of $G$. However, we can construct a no-bend drawing of $G$ if $H\left(x_{3}\right)$ has another appropriate no-bend drawing.

We give an illustrative example in Figure 8 and omit the details of the proof. $G$ has no polar drawing with the poles corresponding to $x_{p}$ as illustrated in Fig. 8(d). However, $G$ may have a no-bend drawing when one considers some other split pair as poles. We therefore consider an SPQ-tree $\mathcal{T}^{\prime}$ of $G$ with $x_{b}$ as the root, as illustrated in Fig. 8(f), where $x_{3}, x_{4}$ and $x_{5}$ are the children of $x_{b}$. Each of $D\left(x_{4}\right)$ and $D\left(x_{5}\right)$ remains same as one obtained for the SPQ-tree $\mathcal{T}$. Considering $\mathcal{T}^{\prime}, D\left(x_{3}\right)$ is a diagonal drawing $D^{\prime}$. We can thus find a no-bend drawing of $G$ by recursively applying Algorithm Biconnected-Draw regarding $D\left(x_{3}\right), D\left(x_{4}\right)$ and $D\left(x_{5}\right)$ as new $D\left(x_{1}\right), D\left(x_{2}\right)$ and $D\left(x_{3}\right)$, respectively. (Figure 8 (h) shows that $G$ has a no-bend polar drawing with the poles corresponding to root $x_{b}$.) If we cannot draw a no-bend orthogonal drawing of $G$ by repeating the operation above, then $G$ does not have a no-bend drawing.

Thus Algorithm Biconnected-Draw finds a no-bend drawing of $G$ if $G$ has. One can efficiently implement Algorithm Biconnected-Draw so that it takes time $O(n)$. The details are omitted in this extended abstract.

Theorem 1. Let $G$ be a biconnected series-parallel graph of the maximum degree three. Then Algorithm Biconnected-Draw finds a no-bend drawing of $G$ in time $O(n)$ whenever $G$ has, where $n$ is the number of vertices of $G$. 


\section{Conclusions}

In this paper, we gave a linear-time algorithm to find a no-bend drawing of a biconnected series-parallel graph $G$ of maximum degree at most three. We also gave an algorithm to find a no-bend drawing of a series-parallel graph $G$ which is not always biconnected. However, the algorithm is omitted in this extended abstract due to page limitation. It is left as a future work to find a bend-minimum drawing of series-parallel graphs and to find a linear-time algorithm for a larger class of planar graphs.

\section{References}

[BEGKLM04] F. Brandenburg, D. Eppstein, M. T. Goodrich, S. Kobourov, G. Liotta and P. Mutzel, Selected open problems in graph drawings, Proc. of GD '03, Lect, Notes in Computer Science, 1912, pp. 515-539, 2004.

[DLV98] G. Di Battista, G. Liotta and F. Vargiu, Spirality and optimal orthogonal drawings, SIAM J. Comput., 27(6), pp. 1764-1811, 1998.

[DT96] G. Di Battista and R. Tamassia, On-line planarity testing, SIAM J. Comput., 25(5), pp. 956-997, 1996.

[GL99] A. Garg and G. Liotta, Almost bend-optimal planar orthogonal drawings of biconnected degree-3 planar graphs in quadratic time, Proc. of GD '99, Lect. Notes in Computer Science, 1731, pp. 38-48, 1999.

[GT01] A. Garg and R. Tamassia, On the computational complexity of upward and rectilinear planarity testing, SIAM J. Comput., 31(2), pp. 601-625, 2001.

[NR04] T. Nishizeki and M. S. Rahman, Planar Graph Drawing, World Scientific, Singapore, 2004.

[REN05] M. S. Rahman, N. Egi and T. Nishizeki, No-bend orthogonal drawings of subdivisions of planar triconnected cubic graphs, IEICE Trans. Inf. \& Syst., E88-D (1), pp.23-30, 2005.

[RN02] M. S. Rahman and T. Nishizeki, Bend-minimum orthogonal drawings of plane 3-graphs, Proc. of WG '02, Lect. Notes in Computer Science, 2573, pp. 265-276, 2002.

[RNN03] M. S. Rahman, M. Naznin and T. Nishizeki, Orthogonal drawings of plane graphs without bends, Journal of Graph Alg. and Appl., 7(4), pp. 335-362, 2003.

[RNN99] M.S. Rahman, S. Nakano and T. Nishizeki, A linear algorithm for bend-optimal orthogonal drawings of triconnected cubic plane graphs, Journal of Graph Alg. and Appl., http://www.cs.brown.edu/publications/jgaa/, 3(4), pp. 31-62, 1999.

[T87] R. Tamassia, On embedding a graph in the grid with the minimum number of bends, SIAM J. Comput., 16, pp. 421-444, 1987. 